\title{
Challenges for the utilization of vaccines against arthritogenic alphaviruses
}
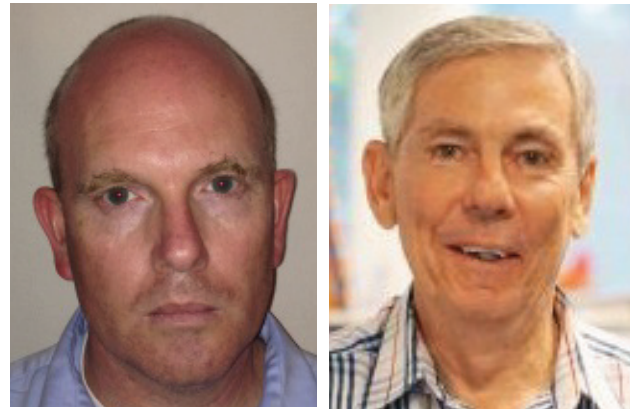

David WC Beasley ${ }^{* 1}$ \& John G Aaskov²

\begin{abstract}
"Many of the 'Old World'
alphaviruses ... that are

transmitted by mosquitoes

(o'nyong'nyong, chikungunya, Ross River, Barmah Forest and Sindbis) have been associated with fever, arthritis and myalgia
\end{abstract} in humans."

First draft submitted: 1 February 2016; Accepted for publication: 2 February 2016; Published online: 23 March 2016

Many of the 'Old World' alphaviruses (family: Togaviridae, genus: Alphavirus) that are transmitted by mosquitoes (o'nyong'nyong, chikungunya, Ross River, Barmah Forest and Sindbis) have been associated with fever, arthritis and myalgia in humans. Whether the ratio of clinical to subclinical infection with each virus is the same and whether the prevalence of arthritis following infection is similar also remains unclear because of the dearth of laboratory facilities able to confirm infection in the localities where many of these infections occur. However, prolonged arthritic symptoms have been observed in confirmed infections with all these viruses [1].

Chikungunya virus (CHIKV) was first isolated in Tanzania, Africa in 1953 and was associated with periodic large epidemics of disease in Africa and Asia throughout the 20th century. Epidemics of CHIKV infection are associated with high attack rates and a relatively low rate of subclinical infections, meaning they are often explosive in nature but self-limiting [2]. Ross River virus (RRV) is endemic in Australia, causing more than 9000 cases of epidemic polyarthritis in 2015 and it was responsible for an epidemic that swept Fiji, Samoa, Tonga and the Cook Islands in the Pacific in 1979-1980 resulting in tens of thousands of cases of clinical disease. After an absence of more than 30 years, RRV has become endemic in Fiji [3]. Strains of Sindbis virus (SINV) (Ockelbo, Pogosta, Karelian fever) have been responsible for large outbreaks of prolonged polyarthralgia in northern Europe and Russia [4].

The US Army developed candidate inactivated and live attenuated vaccines against $\mathrm{CHIKV}$ as part of their biodefence program between 1962 and 2011 [5]. Although the live attenuated candidate reached Phase II clinical trials, development was discontinued due to the anticipated difficulty in

\section{KEYWORDS}

- alphaviruses • chikungunya virus

- Ross River virus • vaccines

"The re-emergence of chikungunya over the past decade prompted a renewed interest in the development of candidate vaccines, therapies and diagnostic assays.” 


\section{“The development of inactivated and other vaccines against arthritogenic alphaviruses is technically simple and the relative ease of production has been demonstrated."}

performing field efficacy studies when outbreaks were unpredictable, and challenges in sustaining budgets for the program. In 2005-2006, large epidemics involving hundreds of thousands of cases of CHIKV polyarthritis occurred in Kenya and on La Reunion and some other Indian Ocean islands. These outbreaks were associated with the emergence of a new variant, designated the Indian Ocean lineage (IOL), characterized by adaptive mutations in the E1 and E2 proteins that enhanced dissemination in Aedes albopictus mosquitoes [6]. During the outbreak on La Reunion Island, several novel and potentially fatal disease presentations were identified in a small number of patients, predominantly neonates, the elderly and individuals with significant comorbidities. Subsequently, this IOL variant was transported globally by infected travelers and was responsible for $>1$ million cases in southern India, smaller outbreaks in other south/southeast Asian countries, and even some autochthonous cases in southern Europe. In late 2013, CHIKV was detected in the Caribbean and has subsequently spread widely through central and South America resulting in almost 1.8 million suspected and 57,170 confirmed cases to January 2016 [7]. During this time frame, outbreaks of CHIKV infection also have occurred in the south Pacific. These recent outbreaks have been associated with Asian or African genotype viruses rather than the IOL variant that emerged in 2005.The re-emergence of CHIKV over the past decade prompted a renewed interest in the development of candidate vaccines, therapies and diagnostic assays. More than 20 different candidate vaccines utilizing a wide range of platforms/approaches and potential single or multidose immunization schedules have been developed, of which a handful have entered, or soon will enter, clinical trials [8,9]. All of these candidates aim to stimulate neutralizing antibodies against the E1 and E2 structural proteins on the virion. A multisite Phase II trial (ClinicalTrials. gov: NCT02562482) of a candidate virus-like particle vaccine developed by the US NIH began in the Caribbean in October 2015 and is scheduled to run until December 2017. This trial will assess safety and immunogenicity of the vaccine in healthy adults, evaluate additional parameters of humoral and cell-mediated immunity following vaccination and compare the incidence of CHIKV infection in vaccinated versus unvaccinated participants. Although the study areas are in countries/territories with significant
CHIKV transmission in 2014 and/or 2015, the reported occurrence of new infections in all is currently low. Given the unpredictable nature of outbreaks of CHIKV infection and the lack of robust epidemiological information regarding the ongoing burden of disease in postepidemic or endemic areas, the size and duration of studies necessary to demonstrate efficacy cannot be readily determined. An alternative approach might be via definition of a correlate of protection, possibly by determining levels of neutralizing antibodies associated with long-lasting protection following natural $\mathrm{CHIKV}$ infection.

An inactivated RRV vaccine developed in Australia faced similar challenges [10]. The decision to develop an inactivated vaccine was influenced by the expectation that it was likely to be much less expensive to demonstrate safety with a killed than a live attenuated vaccine. The initial vaccine was derived using binary ethyleneimine (BEI) to inactivate the virus because this agent acted on viral nucleic acid and would destroy infectivity without effecting immunogenicity and there was an extensive literature relating to its use in veterinary settings. Subsequently, it was decided to develop a double inactivated vaccine, employing formalin as one of the inactivating agents, based on the lack of any protocol that would be acceptable to licensing agencies that demonstrated the complete removal of BEI at the conclusion of the inactivation process. Baxter, with whom a cooperative research and development agreement (CRADA) had been signed to develop a human vaccine, had extensive experience producing safe formalin inactivated vaccines and UV irradiation would be an acceptable substitute for BEI to inactivate viral RNA [11].

As is the case with CHIKV vaccines, a major challenge was to demonstrate vaccine efficacy, given the relatively low rates and variable distributions of clinical cases in Australia each year. The size of the potential market for a RRV vaccine also would not justify the cost of a standard efficacy study. However, the Australian national regulatory authority (the Therapeutic Goods Administration) agreed to consider correlates of protection as a substitute for measurements of efficacy. There have been no confirmed examples of an epidemic polyarthritis patient (clinical RRV infection) experiencing a second clinical infection or of someone with a second infection developing clinical symptoms, suggesting that a natural infection provides life-long protection against a subsequent clinical infection with 
RRV. It was possible to locate, and obtain serum from, a panel of former epidemic polyarthritis patients who had participated in research studies decades previously and to determine the levels of residual neutralizing antibody in these subjects [12]. These were regarded as the minimum levels that an efficacious vaccine would need to elicit. These data were supported by active and passive immunization studies with virus challenge in laboratory animals [13].

The RRV vaccine has completed Phase III trials and is safe and immunogenic and elicits levels of neutralizing antibody comparable to a natural infection [14]. It is possible that the vaccine could be licensed in Australia if a sponsor agreed to postmarketing surveillance. The challenges for the roll out of a RRV vaccine include: the virus does not kill people; a limited market at present (adult Australians and tourists); the likely cost of the vaccine against a saving of only approximately AUD \$20 million per year in direct and associated medical costs; a reluctance to consider the social costs of prolonged arthritis; and a growing immunization apathy and antivaccination lobby. Similar issues related to the commercial viability of CHIKV vaccines may also pose barriers to successful licensure of candidates currently under development and evaluation. In the absence of an ongoing, dramatic and clearly apparent burden of disease, public and political attention and associated financial support necessary to move promising candidates forward is likely to wane in the face of other emerging public health priorities. As the most obvious example, the recent emergence of Zika virus in South America and its potential association with microcephaly and Guillain-Barré syndrome has rapidly pushed CHIKV from the headlines. Importantly for CHIKV, the capacity

\section{References}

1 Suhrbier A, Jaffar-Bandjee MC, Gasque P. Arthritogenic alphaviruses - an overview. Nat. Rev. Rheumatol. 8(7), 420-429 (2012).

2 Weaver SC, Lecuit M. Chikungunya virus and the global spread of a mosquito-borne disease. N. Engl. J. Med. 372(13), 1231-1239 (2015).

3 Lau C, Weinstein P, Slaney D. Imported cases of Ross River virus disease in New Zealand - a travel medicine perspective. Travel Med. Infect. Dis. 10(3), 129-134 (2012).

to develop and manufacture vaccines exists in some endemic countries, even if the apparent threat of the virus to more affluent nations wanes.

The development of inactivated and other vaccines against arthritogenic alphaviruses is technically simple and the relative ease of production has been demonstrated. For CHIKV, an abundance of promising vaccine candidates exists and developers are seeking a path forward. The two major challenges to eventual licensure and utilization of CHIKV and RRV vaccines are the cost and practicality of clinical trials to demonstrate efficacy and the ability or willingness of the market to pay for a vaccine against a disease that is unlikely to kill them. Two strong justifications for the development and utilization of these vaccines are the significant social costs of a prolonged and debilitating arthritis, particularly in those with young families, and a 'duty of care' on the part of employers whose staff are required to work in settings where they have extensive exposure to mosquitoes likely to be carrying these viruses.

\section{Financial \& competing interests disclosure}

JG Aaskov's employer, the Queensland University of Technology, had a cooperative research and development agreement with Immuno and subsequently Baxter during the development of the Ross River virus vaccine. JG Aaskov received no direct financial support other than to attend Ross River virus vaccine project meetings. The authors have no other relevant affliations or financial involvement with any organization or entity with a financial interest in or financial conflict with the subject matter or materials discussed in the manuscript apart from those disclosed.

No writing assistance was utilized in the production of this manuscript.

4 Laine M, Luukkainen R, Toivanen A. Sindbis viruses and other alphaviruses as cause of human arthritic disease. J. Intern. Med. 256(6), 457-471 (2004).

5 Hoke CH Jr, Pace-Templeton J, Pittman P et al. US military contributions to the global response to pandemic chikungunya. Vaccine 30(47), 6713-6720 (2012).

6 Weaver SC, Forrester NL. Chikungunya: evolutionary history and recent epidemic spread. Antiviral Res. 120, 32-39 (2015).

7 Pan American Health Organization. Pan American Health Organization program - chikungunya (2016). www.paho.org/hq/? Itemid = 40931
8 Weaver SC, Osorio JE, Livengood JA, Chen $\mathrm{R}$, Stinchcomb DT. Chikungunya virus and prospects for a vaccine. Expert Rev. Vaccines 11(9), 1087-1101 (2012).

9 Smalley C, Erasmus JH, Chesson CB, Beasley DWC. Status of research and development of vaccines for chikungunya. Vaccine (2016) (In Press).

10 Yu S, Aaskov JG Development of a candidate vaccine against Ross River virus infection. Vaccine 12(12), 1118-1124 (1994).

11 Aichinger G, Ehrlich HJ, Aaskov JG et al. Safety and immunogenicity of an inactivated whole virus vero cell-derived Ross 
River virus vaccine: a randomized trial. Vaccine 29(50), 9376-9384 (2011).

12 Kistner O, Barrett N, Bruhmann A et al. The preclinical testing of a formaldehyde inactivated Ross River virus vaccine designed for use in humans. Vaccine 25(25), 4845-4852 (2007).
13 Holzer GW, Coulibaly S, Aichinger G et al. Evaluation of an inactivated Ross River virus vaccine in active and passive mouse immunization models and establishment of a correlate of protection. Vaccine 29(24), 4132-4141 (2011).
14 Wressnigg N, Van Der Velden MV, Portsmouth D et al. An inactivated Ross River virus vaccine is well tolerated and immunogenic in an adult population in a randomized Phase 3 trial. Clin. Vaccine Immunol. 22(3), 267-273 (2015). 